\title{
Spin-Orbit Coupling and Time-Reversal Symmetry in Quantum Gates
}

\author{
D. Stepanenko and N.E. Bonesteel \\ Department of Physics and National High Magnetic Field Laboratory, Florida State University, Tallahassee, FL 32310 \\ D.P. DiVincenzo and G. Burkard \\ IBM Research Division, T.J. Watson Research Center, Yorktown Heights, NY 10598 \\ Daniel Loss \\ Department of Physics and Astronomy, University of Basel, Klingelbergstrasse 82, CH-4056 Basel, Switzerland
}

\begin{abstract}
We study the effect of spin-orbit coupling on quantum gates produced by pulsing the exchange interaction between two single electron quantum dots. Spin-orbit coupling enters as a small spin precession when electrons tunnel between dots. For adiabatic pulses the resulting gate is described by a unitary operator acting on the four-dimensional Hilbert space of two qubits. If the precession axis is fixed, time-symmetric pulsing constrains the set of possible gates to those which, when combined with single qubit rotations, can be used in a simple CNOT construction. Deviations from time-symmetric pulsing spoil this construction. The effect of time asymmetry is studied by numerically integrating the Schrödinger equation using parameters appropriate for GaAs quantum dots. Deviations of the implemented gate from the desired form are shown to be proportional to dimensionless measures of both spin-orbit coupling and time asymmetry of the pulse.
\end{abstract}

\section{INTRODUCTION}

A promising proposal for building a solid-state quantum computer is based on the notion of using electron spins trapped in quantum dots as qubits. ${ }^{1}$ In such a device, two-qubit quantum gates would be carried out by turning on and off the exchange interaction between spins on neighboring dots through suitable pulsing of gate voltages.

When performing such a quantum gate, if nonadiabatic errors $^{2-4}$ can be safely ignored, ${ }^{5}$ both the initial and final states of the two dots will be in the four-dimensional Hilbert space of two qubits. In the absence of spin-orbit coupling, and neglecting the dipolar interaction between spins, the unitary transformation resulting from such a pulsed exchange gate will necessarily have the form

$$
U=\exp -i \lambda \mathbf{S}_{A} \cdot \mathbf{S}_{B},
$$

where $\lambda$ is a dimensionless measure of the pulse strength. This simple isotropic form is a consequence of symmetry - if spin and space decouple exactly, as they do in the nonrelativistic limit, then the system is perfectly isotropic in spin space. Up to an irrelevant overall phase the gates (1) are the most general unitary operators with this symmetry acting on a two-qubit Hilbert space.

These isotropic exchange gates are useful for quantum computation. In conjunction with single qubit rotations, they can be used in a simple construction of a controlled-not (CNOT) gate. ${ }^{1}$ It has also been shown that, even without single qubit rotations, isotropic exchange gates can be used for universal quantum computing with proper encoding of logical qubits. ${ }^{6,7}$

When the effects of spin-orbit coupling are included, well-isolated single electron dots will have a two-fold
Kramers degeneracy and so can still be used as qubits. However, when carrying out a quantum gate the total spin will no longer be a good quantum number. As a result there will inevitably be corrections to the isotropic exchange gates (1). Motivated by this fact, a number of authors have considered anisotropic gates of the form

$$
\begin{aligned}
U=\exp -i \lambda & \left(\mathbf{S}_{A} \cdot \mathbf{S}_{B}+\boldsymbol{\beta} \cdot\left(\mathbf{S}_{A} \times \mathbf{S}_{B}\right)\right. \\
& +\gamma\left(\mathbf{S}_{A} \cdot \mathbf{S}_{B}-\left(\hat{\boldsymbol{\beta}} \cdot \mathbf{S}_{A}\right)\left(\hat{\boldsymbol{\beta}} \cdot \mathbf{S}_{B}\right)\right),
\end{aligned}
$$

and shown that they have several useful properties. For example, in Ref. 8 it was shown that the CNOT construction of Ref. 1 is robust against anisotropic corrections of the form appearing in (2). It has also been shown that, when combined with a controllable Zeeman splitting, the gates (2) form a universal set. ${ }^{9}$

The anisotropic terms which appear in (2) are not the most general corrections to (1) which can occur when carrying out an exchange gate in the presence of spin-orbit coupling. It is therefore important to ask under what conditions these corrections can be restricted to have this desired form. The key observation motivating the present work is that, up to an irrelevant overall phase, the gates (2) are the most general two-qubit quantum gates which are both axially symmetric, i.e. symmetric under rotations about an axis parallel to the vector $\boldsymbol{\beta}$ in spin space, and symmetric under time reversal $\left(\mathbf{S}_{\mu} \rightarrow-\mathbf{S}_{\mu}\right.$, $\mu=A, B)$. It follows that if these symmetries can be maintained throughout the gate operation, and provided nonadiabatic errors can be neglected, the resulting quantum gate is guaranteed to have the form (2). Of course symmetry alone cannot determine the values of $\lambda, \boldsymbol{\beta}$ and $\gamma$. However, in practice we envision these parameters will 
be determined through experimental calibration rather than microscopic calculation. Therefore we emphasize symmetry as a useful guiding principle.

In this paper we study the effect of spin-orbit coupling on exchange-based quantum gates. For concreteness we consider a system of two single-electron quantum dots in GaAs. The contribution of spin-orbit coupling to the exchange interaction between localized spins in GaAs has been studied by Kavokin ${ }^{10}$ within the Heitler-London approximation, and by Gor'kov and Krotkov ${ }^{11}$ who derived the exact asymptotic exchange interaction between hydrogen-like bound states at large separation.

Here we follow Ref. 2 and work within the HundMulliken approximation, keeping one orbital per dot, and allowing double occupancy. In this approximation, the effect of spin-orbit coupling is to induce a small spin precession whenever an electron tunnels from one dot to another. The Hamiltonian governing the two-dot system is therefore axially symmetric in spin space with the symmetry axis being the precession axis of the spin. If the direction of the precession axis does not change while the gate is being pulsed, then the resulting quantum gate will also be axially symmetric.

An additional useful symmetry principle, first suggested in Ref. 12, is that any time-dependent Hamiltonian $H_{P}(t)$ which is time-reversal symmetric at all times $t$, and which is then pulsed in a time-symmetric way $\left(H_{P}(t)=H_{P}(-t)\right)$ will lead to a gate which can be described in terms of an effective time-independent Hamiltonian $H$ which is also time-reversal symmetric. Here we give a proof of this result.

Taken together these two results imply that, within the Hund-Mulliken approximation, if the spin-orbit precession axis is fixed and nonadiabatic errors can be ignored, the unitary transformation produced by pulsing the exchange interaction between two quantum dots will necessarily have the desired form (2) provided the gate is pulsed in a time-symmetric way.

This paper is organized as follows. In Sec. II we derive the Hund-Mulliken Hamiltonian for a double quantum dot system in the presence of spin-orbit coupling. In Sec. III we develop an effective spin Hamiltonian description which can be applied to pulsing our double dot system, and we review the robust CNOT construction of Ref. 8. The implications of time-symmetric pulsing are then studied in Sec. IV, and in Sec. V we present numerical results showing the effect of small time asymmetry of the pulse. Finally, in Sec. VI we summarize the results of the paper.

\section{HUND-MULLIKEN HAMILTONIAN}

We consider a system of two laterally confined quantum dots with one electron in each dot. For concreteness we assume the dots are formed in a two-dimensional electron gas (2DEG) realized in a GaAs heterostructure.
The system is modeled by the Hamiltonian

$$
H=T+C+H_{S O} .
$$

Here $T+C$ is the Hamiltonian studied in Ref. 2, where $T=\sum_{i} h_{i}$ with

$$
h_{i}=\frac{1}{2 m}\left(\mathbf{p}_{i}-\frac{e}{c} \mathbf{A}\left(\mathbf{r}_{i}\right)\right)^{2}+V\left(\mathbf{r}_{i}\right),
$$

and $C=e^{2} / \epsilon\left|\mathbf{r}_{1}-\mathbf{r}_{2}\right|$ is the Coulomb repulsion between electrons. We take the 2DEG the dots are formed in to lie in the $x y$ plane, and for GaAs we take $m=0.067 m_{e}$ and $\epsilon=13.1$. For completeness we include a vector potential $\mathbf{A}=(-y, x, 0) B / 2$ which couples the orbital motion of the electrons to a uniform magnetic field $\mathbf{B}=B \hat{\mathbf{z}}$. We will see in Sec. III that this orbital coupling does not affect any of our arguments based on time-reversal symmetry, while a nonzero Zeeman coupling does.

As in Ref. 2 lateral confinement of the dots is modeled by the double-well potential,

$$
V(x, y)=\frac{m \omega_{0}^{2}}{2}\left(\frac{1}{4 a^{2}}\left(x^{2}-a^{2}\right)^{2}+y^{2}\right) .
$$

This potential describes two quantum dots sitting at the points $(x, y)=( \pm a, 0)$. In the limit of large separation the dots decouple into two harmonic wells with frequency $\omega_{0}$.

Spin-orbit coupling enters the Hamiltonian through the term

$$
H_{S O}=\sum_{i=1,2} \boldsymbol{\Omega}\left(\mathbf{k}_{i}\right) \cdot \mathbf{S}_{i}
$$

where $\hbar \mathbf{k}=\mathbf{p}-\frac{e}{c} \mathbf{A}$. Time-reversal symmetry requires that $\boldsymbol{\Omega}(\mathbf{k})$ is an odd function of $\mathbf{k}, \boldsymbol{\Omega}(\mathbf{k})=-\boldsymbol{\Omega}(-\mathbf{k})$. Thus $\boldsymbol{\Omega}$ is nonzero only in the absence of inversion symmetry.

For definiteness, we take the 2DEG in which the dots are formed to lie in the plane perpendicular to the [001] structural direction, which then points along the $z$-axis. However, we allow the $x$-axis, which is parallel to the displacement vector of the two dots, to have any orientation with the respect to the [100] and [010] structural axes. To describe the dependence of $\boldsymbol{\Omega}$ on $\mathbf{k}$ it is then convenient to introduce unit vectors $\hat{\mathbf{e}}_{[110]}$ and $\hat{\mathbf{e}}_{[\overline{1} 10]}$ which point in the [110] and [110] structural directions, respectively, and define $k_{[110]}=\mathbf{k} \cdot \hat{\mathbf{e}}_{[110]}$ and $k_{[\overline{1} 10]}=\mathbf{k} \cdot \hat{\mathbf{e}}_{[\overline{1} 10]} \cdot$ We then have, following Kavokin, ${ }^{10}$

$$
\boldsymbol{\Omega}(\mathbf{k}) \simeq\left(f_{D}-f_{R}\right) k_{[110]} \hat{\mathbf{e}}_{[\overline{1} 10]}+\left(f_{D}+f_{R}\right) k_{[\overline{1} 10]} \hat{\mathbf{e}}_{[110]} .
$$

Here $f_{D}$ is the Dresselhaus contribution ${ }^{13,14}$ due to the bulk inversion asymmetry of the zinc-blende crystal structure of GaAs, and $f_{R}$ is the Rashba contribution ${ }^{15}$ due to the inversion asymmetry of the quantum well used to form the 2DEG. These quantities depend on details of the 2DEG confining potential and so will vary from system to system. 
It was pointed out in Ref. 16 that $H_{S O}$ has a special symmetry when $f_{D}= \pm f_{R}$. This can be seen directly from (7). When $f_{D}=f_{R}\left(f_{D}=-f_{R}\right)$ the direction of $\boldsymbol{\Omega}$ is independent of $\mathbf{k}$ and is fixed to be parallel to $\hat{\mathbf{e}}_{[110]}$ $\left(\hat{\mathbf{e}}_{[\overline{1} 10]}\right)$. The full Hamiltonian (3) is then invariant under rotations in spin space about this axis. We will see below that this special case has a number of attractive features.

In the limit of decoupled dots, and ignoring spin-orbit coupling, the single electron ground states will be the Fock-Darwin ground states centered at $(x, y)=( \pm a, 0)$,

$$
\phi_{ \pm a}(x, y)=\sqrt{\frac{m \omega}{\pi \hbar}} e^{-m \omega\left((x \mp a)^{2}+y^{2}\right) / 2 \hbar} e^{ \pm i a y / 2 l_{B}^{2}} .
$$

Here $\omega^{2}=\omega_{0}^{2}+\omega_{L}^{2}$ is the frequency of the magnetically squeezed oscillator where $\omega_{L}=e B / 2 m c$ is the Larmor frequency and $l_{B}=\sqrt{\hbar c / e B}$ is the magnetic length. In zero magnetic field, the size of these wave functions is set by the effective "Bohr radius" $a_{B}=\sqrt{\hbar / m \omega_{0}}$.

The Fock-Darwin states can be orthogonalized to obtain the Wannier states

$$
\begin{aligned}
\Phi_{A} & =\frac{1}{\sqrt{1-2 S g-g^{2}}}\left(\phi_{a}-g \phi_{-a}\right), \\
\Phi_{B} & =\frac{1}{\sqrt{1-2 S g-g^{2}}}\left(\phi_{-a}-g \phi_{a}\right),
\end{aligned}
$$

where $S=\left\langle\phi_{-a} \mid \phi_{a}\right\rangle$ and $g=\left(1-\sqrt{1-S^{2}}\right) / S$. We can then introduce second quantized operators $c_{A_{\alpha}}^{\dagger}\left(c_{A_{\alpha}}\right)$ and $c_{B \alpha}^{\dagger}\left(c_{B \alpha}\right)$ which create (annihilate) electrons in the states $\Phi_{A}$ and $\Phi_{B}$ with spin $\alpha=\uparrow, \downarrow$.

In the Hund-Mulliken approximation we keep one orbital per dot and allow for double occupancy. This amounts to restricting the full Hilbert space of the problem to the six-dimensional Hilbert space spanned by the states

$$
\begin{aligned}
\left|S_{1}\right\rangle & =\frac{1}{\sqrt{2}}\left(c_{A \uparrow}^{\dagger} c_{B \downarrow}^{\dagger}-c_{A \downarrow}^{\dagger} c_{B \uparrow}^{\dagger}\right)|0\rangle, \\
\left|S_{2}\right\rangle & =\frac{1}{\sqrt{2}}\left(c_{A \uparrow}^{\dagger} c_{A \downarrow}^{\dagger}+c_{B \downarrow}^{\dagger} c_{B \uparrow}^{\dagger}\right)|0\rangle, \\
\left|S_{3}\right\rangle & =\frac{1}{\sqrt{2}}\left(c_{A \uparrow}^{\dagger} c_{A \downarrow}^{\dagger}-c_{B \downarrow}^{\dagger} c_{B \uparrow}^{\dagger}\right)|0\rangle, \\
\left|T_{-}\right\rangle & =c_{A \downarrow}^{\dagger} c_{B \downarrow}^{\dagger}|0\rangle, \\
\left|T_{0}\right\rangle & =\frac{1}{\sqrt{2}}\left(c_{A \uparrow}^{\dagger} c_{B \downarrow}^{\dagger}+c_{A \downarrow}^{\dagger} c_{B \uparrow}^{\dagger}\right)|0\rangle, \\
\left|T_{+}\right\rangle & =c_{A \uparrow}^{\dagger} c_{B \uparrow}^{\dagger}|0\rangle .
\end{aligned}
$$

In terms of second quantized operators, the HundMulliken Hamiltonian acting in this space, up to an irrelevant overall additive constant, can be written

$$
\begin{aligned}
H_{H M}= & \sum_{\alpha, \beta=\uparrow, \downarrow}-\left(c_{A \alpha}^{\dagger}\left(t_{H} \delta_{\alpha \beta}+i \mathbf{P} \cdot \boldsymbol{\sigma}_{\alpha \beta}\right) c_{B \beta}+H . c .\right) \\
& +V\left(\mathbf{S}_{A} \cdot \mathbf{S}_{B}+3 / 4\right) \\
& +U_{H}\left(n_{A \uparrow} n_{A \downarrow}+n_{B \uparrow} n_{B \downarrow}\right) .
\end{aligned}
$$

Here

$$
\mathbf{S}_{\mu}=\frac{1}{2} \sum_{\alpha, \beta=\uparrow, \downarrow} c_{\mu_{\alpha}}^{\dagger} \boldsymbol{\sigma}_{\alpha \beta} c_{\mu_{\beta}}
$$

is the spin operator on site $\mu=A, B$,

$$
V=\left\langle S_{1}|C| S_{1}\right\rangle-\langle T|C| T\rangle
$$

is the ferromagnetic direct exchange,

$$
U_{H}=\left\langle S_{2}|C| S_{2}\right\rangle-\left\langle S_{1}|C| S_{1}\right\rangle
$$

is the Coulomb energy cost of doubly occupying a dot, and

$$
t_{H}=\left\langle\Phi_{A}|h| \Phi_{B}\right\rangle
$$

is the interdot tunneling amplitude.

The only contribution from spin-orbit coupling is the matrix element

$$
i \mathbf{P}=\left\langle\Phi_{A}|\boldsymbol{\Omega}(\mathbf{k})| \Phi_{B}\right\rangle=\left\langle\Phi_{A}\left|\left(p_{x}-\frac{e}{c} A_{x}\right) / \hbar\right| \Phi_{B}\right\rangle \boldsymbol{\eta},
$$

where

$$
\boldsymbol{\eta}=\left(f_{D}-f_{R}\right) \cos \theta \hat{\mathbf{e}}_{[\overline{1} 10]}+\left(f_{D}+f_{R}\right) \sin \theta \hat{\mathbf{e}}_{[110]} .
$$

Here $\theta$ is the angle the $x$-axis makes with the [110] structural direction. This term introduces a small spin precession about an axis parallel to $\mathbf{P}$ through an angle $\phi=2 \arctan \left(P / t_{H}\right)$ when an electron tunnels between dots.

It is convenient to express the spin-orbit matrix element as $\mathbf{P}=s \mathbf{l}_{S O}$ where

$$
s=\frac{\sqrt{\left(f_{D}-f_{R}\right)^{2} \cos ^{2} \theta+\left(f_{D}+f_{R}\right)^{2} \sin ^{2} \theta}}{a_{B} \hbar \omega_{0}}
$$

is a dimensionless measure of the strength of spin-orbit coupling. As stated above, $f_{D}$ and $f_{R}$ depend on details of the potential confining the electron to the 2DEG. Thus $\theta, f_{D}$ and $f_{R}$ are all parameters that in principle can be engineered to control the value of $s$. For example, if $\theta=0$ then $s=\left|f_{D}-f_{R}\right| /\left(a_{B} \hbar \omega_{0}\right)$. Thus, for this orientation of the dots, if it is possible to design a system in which $f_{D}=f_{R}, s$ can be made to vanish. Even if such perfect cancellation cannot be achieved, minimizing the difference $f_{D}-f_{R}$ will reduce $s$.

In what follows we leave $s$ as a free parameter. We estimate that for GaAs quantum dots $s<0.1$ for typical parameters. ${ }^{10}$ The remaining contribution to the matrix element $\mathbf{P}$ is then

$$
\mathbf{l}_{S O}=\frac{\hbar \omega_{0}}{2} \frac{1-g^{2}}{1-2 S g+g^{2}} \frac{d}{b} e^{-d^{2} b\left(2-1 / b^{2}\right)} \hat{\boldsymbol{\eta}},
$$

where $d=a / a_{B}$ is a dimensionless measure of the distance between dots, $b=\sqrt{1+\omega_{L}^{2} / \omega_{0}^{2}}$, and $\hat{\boldsymbol{\eta}}=\boldsymbol{\eta} / \eta$. The 
geometry of our model system is shown schematically in Fig. 1.

In what follows we envision pulsing quantum gates by varying the distance $d$ between dots as a function of time. In doing this, we will assume that throughout the pulse the values of $f_{D}$ and $f_{R}$ do not change. If this is the case $s$ will be constant and all of the time dependence of $\mathbf{P}$ will be due to $\mathbf{l}_{S O}$. In addition, the direction of the vector $\mathbf{P}$ will not change as a function of time. The Hamiltonian $H_{H M}$ will therefore be invariant under rotations in spin space about a single fixed axis parallel to $\mathbf{P}$ throughout the pulse. We will refer to such a pulse as having axial symmetry.



FIG. 1. Sketch of the GaAs double quantum dot system considered in this paper. There is one electron per dot, and the dot separation is $2 a$. The dots are taken to lie in the plane perpendicular to both the [001] axis and an applied magnetic field $B$. The displacement of the dots makes an angle $\theta$ with the [110] axis. Due to spin-orbit coupling electron spins precess about an axis parallel to $\mathbf{P}$ when tunneling between dots.

It is important to note that this axial symmetry is approximate. In general $f_{D}$ and $f_{R}$ will depend on time as the gate is pulsed, though in principle the system can be engineered to minimize this effect. Also, for general $f_{D}$ and $f_{R}$ the appearance of only one vector in spin space is a consequence of restricting the Hilbert space to one orbital per dot. If more orbitals are included then more spin-orbit matrix elements will appear in the Hamiltonian, corresponding typically to different spin-precession axes, thus breaking the axial symmetry. However, as shown above, if $f_{D}= \pm f_{R}$ then the full Hamiltonian (3) is axially symmetric - thus for this special case all spin precession axes will be parallel and axial symmetry will not just be an artifact of the Hund-Mulliken approximation. In Sec. V we discuss the effect deviations from axial symmetry will have on our results.

Given an axially symmetric pulse, it is convenient to take the $z$-axis in spin space to be parallel to $\mathbf{P}$. For this choice, the states $\left|T_{+}\right\rangle$and $\left|T_{-}\right\rangle$decouple, each having energy $V$.

Another useful symmetry of $H_{H M}$ is invariance under $c_{A \alpha} \rightarrow c_{B,-\alpha}$ and $c_{B \alpha} \rightarrow c_{A,-\alpha}$. This transformation changes the sign of the states $\left|S_{1}\right\rangle,\left|S_{2}\right\rangle$ and $\left|T_{0}\right\rangle$, while leaving $\left|S_{3}\right\rangle$ invariant. It follows that the state $\left|S_{3}\right\rangle$ also decouples with energy $U_{H}$. The matrix representation of
$H_{H M}$ in the remaining nontrivial $\left|T_{0}\right\rangle,\left|S_{1}\right\rangle,\left|S_{2}\right\rangle$ basis is then

$$
H_{H M}=\left(\begin{array}{ccc}
V & 0 & -2 i P \\
0 & 0 & -2 t_{H} \\
2 i P & -2 t_{H} & U_{H}
\end{array}\right) \text {. }
$$

\section{EFFECTIVE SPIN HAMILTONIAN}

We now consider pulsing the Hamiltonian $H_{H M}$ by varying the distance between the dots, the barrier height, or some combination of the two, in such a way that the two electron spins interact for a finite period of time, but are well separated at the beginning and end of the pulse. We assume the initial state of the system is in the four-dimensional Hilbert space describing two qubits, i.e. the space spanned by the singly occupied states $\left|S_{1}\right\rangle,\left|T_{0}\right\rangle,\left|T_{-}\right\rangle$and $\left|T_{+}\right\rangle$. As the pulse is carried out, the eigenstates of $H_{H M}$ at any given instant in time can be grouped into four low-energy states separated by a gap of order $U_{H}$ from two high-energy states. If the pulse is sufficiently adiabatic on a time scale set by $\sim \hbar / U_{H}$, the amplitude for nonadiabatic transitions which would leave the system in the excited state $\left|S_{2}\right\rangle$ at the end of the pulse can be made negligibly small. ${ }^{5}$ If this condition holds, the final state of the system can also be assumed to be in the four-dimensional Hilbert space of two qubits. We will see that this condition is easily achieved in Sec. V.

One way to theoretically study the effect of such a pulse would be to first reduce $H_{H M}$ to an effective anisotropic spin Hamiltonian acting on the four-dimensional lowenergy Hilbert space and then consider pulsing this effective model. ${ }^{12}$ The problem with this approach is that any such effective spin Hamiltonian will only be valid if the pulse is adiabatic, not only on the time scale $\hbar / U_{H}$, but also on the much longer time scale set by the inverse of the small energy splittings within the low-energy space due to the spin-orbit induced anisotropic terms. However, it is precisely the nonadiabatic transitions induced by these terms which give rise to the quantum gate corrections we would like to compute.

Although we may not be able to define an instantaneous effective spin Hamiltonian during the pulse, we can define one which describes the net effect of a full pulse. This definition amounts to parameterizing the quantum gate produced by the pulse as

$$
U=e^{-i \tau H},
$$

where $U$ acts on the four-dimensional Hilbert space of the initial and final spin states. $H$ is then an effective spin Hamiltonian, i.e. it can be expressed entirely in terms of the spin operators $\mathbf{S}_{A}$ and $\mathbf{S}_{B}$, and $\tau$ is a measure of the pulse duration. Note the definition of $\tau$ is arbitrary because it is the product $\tau H$ which determines $U$. Here, and in the remainder of this paper, we work in units in which $\hbar=1$. 
If we assume exact axial symmetry throughout the pulse, the effective spin Hamiltonian must be invariant under rotations about the $z$-axis in spin space and must also leave the states $\left|T_{+}\right\rangle$and $\left|T_{-}\right\rangle$degenerate. The most general such spin Hamiltonian, up to an irrelevant additive term proportional to the identity operator, is

$$
\begin{aligned}
\tau H(\lambda ; \alpha, \beta, \gamma)=\lambda\left(\mathbf{S}_{A}\right. & \cdot \mathbf{S}_{B}+\frac{\alpha}{2}\left(S_{A z}-S_{B z}\right) \\
+ & \beta\left(S_{A x} S_{B y}-S_{A y} S_{B x}\right) \\
& \left.+\gamma\left(S_{A x} S_{B x}+S_{A y} S_{B y}\right)\right)
\end{aligned}
$$

and we denote the corresponding quantum gate as

$$
U(\lambda ; \alpha, \beta, \gamma)=e^{-i \tau H(\lambda ; \alpha, \beta, \gamma)}
$$

When $\alpha=0$, this is precisely the gate (2) for $\boldsymbol{\beta} \| \hat{\mathbf{z}}$.

The CNOT construction originally proposed in Ref. 1 is based on the sequence of gates

$$
U_{g}=U(\pi / 2 ; 0,0,0) e^{i \pi S_{A z}} U(\pi / 2 ; 0,0,0),
$$

where $U(\pi / 2 ; 0,0,0)=\exp -i\left[(\pi / 2) \mathbf{S}_{A} \cdot \mathbf{S}_{B}\right]$ is a squareroot of swap gate. The CNOT gate is then

$$
U_{C N O T}=e^{i(\pi / 2) S_{A z}} e^{i(\pi / 2) S_{B z}} U_{g} .
$$

Remarkably, it was shown in Ref. 8 that if $\lambda=\pi / 2$ and $\alpha=0$ this construction is robust against the $\beta$ and $\gamma$ corrections, i.e. the gate

$$
U_{g}=U(\pi / 2 ; 0, \beta, \gamma) e^{i \pi S_{A z}} U(\pi / 2 ; 0, \beta, \gamma)
$$

is independent of $\beta$ and $\gamma$.

For completeness, we briefly review the arguments of Ref. 8. Due to axial symmetry, the action of the gate $U(\lambda ; \alpha, \beta, \gamma)$ on the states $\left|T_{+}\right\rangle$and $\left|T_{-}\right\rangle$is trivial and independent of $\alpha, \beta$ and $\gamma$,

$$
U(\lambda ; \alpha, \beta, \gamma)\left|T_{ \pm}\right\rangle=e^{-i \lambda / 4}\left|T_{ \pm}\right\rangle
$$

We can then introduce a pseudospin description of the remaining space, where $\left|S_{1}\right\rangle$ is pseudospin down and $\left|T_{0}\right\rangle$ is pseudospin up. The action of the gate $U(\lambda ; \alpha, \beta, \gamma)$ on this pseudospin space is a simple rotation,

$$
U(\lambda ; \alpha, \beta, \gamma) \Rightarrow e^{i \lambda / 4} e^{-i \mathbf{b} \cdot \boldsymbol{\tau} / 2}
$$

where $\mathbf{b}=\lambda(\alpha, \beta, \gamma+1)$ and the components of $\boldsymbol{\tau}=$ $\left(\tau_{x}, \tau_{y}, \tau_{z}\right)$ are pseudospin Pauli matrices. At the same time, the action of the single qubit rotation entering $U_{g}$ is

$$
e^{i \pi S_{A z}} \Rightarrow i \tau_{x}
$$

Thus to show that the CNOT construction is independent of $\beta$ and $\gamma$ if $\alpha=0$ we need only show that the product

$$
e^{-i \mathbf{b} \cdot \boldsymbol{\tau} / 2} \tau_{x} e^{-i \mathbf{b} \cdot \boldsymbol{\tau} / 2}
$$

is independent of $\beta$ and $\gamma$ if $\alpha=0$. This condition has a simple geometric interpretation. It is the requirement that a rotation about an axis parallel to $\mathbf{b}$, followed by a $180^{\circ}$ rotation about the $x$-axis, and then a repeat of the initial rotation must be equivalent to a simple $180^{\circ}$ rotation about the $x$-axis. This will trivially be the case if the vector $\mathbf{b}=\lambda(\alpha, \beta, \gamma+1)$ lies in the $y z$ plane. Thus, if $\alpha=0$, this condition is satisfied and the CNOT construction is exact. Conversely, if $\alpha \neq 0$ the construction is spoiled.

\section{TIME-REVERSAL SYMMETRY}

In this section we prove the following general result. Any time-dependent Hamiltonian $H_{P}(t)$ which is timereversal symmetric for all $t$, and for which the time dependence is itself symmetric, i.e. $H_{P}\left(t_{0}-t\right)=H_{P}\left(t_{0}+t\right)$ for all $t$, will generate a unitary evolution operator $U=$ $\exp -i \tau H$ where $H$ is a time-independent effective Hamiltonian which is also time-reversal symmetric. We then show that this theorem implies that the parameter $\alpha$, which spoils the CNOT construction described in Sec. III, is equal to zero for time-symmetric pulsing.

The time-reversal operation for any quantum system can be represented by an antiunitary operator $\Theta .{ }^{17}$ An orthonormal basis $\left\{\left|M_{i}\right\rangle\right\}$ for the Hilbert space of this system is then said to be a time-symmetric basis if

$$
\Theta\left|M_{i}\right\rangle=\left|M_{i}\right\rangle
$$

for all $i$.

For any Hamiltonian $H$ acting on a state $\left|M_{i}\right\rangle$ in this basis we can write

$$
H\left|M_{i}\right\rangle=\sum_{j}\left\langle M_{j}|H| M_{i}\right\rangle\left|M_{j}\right\rangle .
$$

Under time reversal $H$ is transformed into $\Theta H \Theta^{-1}$. Using the invariance of the $\left\{\left|M_{i}\right\rangle\right\}$ basis and the antiunitarity of $\Theta$ we can then also write

$$
\begin{aligned}
\Theta H \Theta^{-1}\left|M_{i}\right\rangle & =\Theta H\left|M_{i}\right\rangle \\
& =\Theta \sum_{j}\left\langle M_{j}|H| M_{i}\right\rangle\left|M_{j}\right\rangle \\
& =\sum_{j}\left\langle M_{j}|H| M_{i}\right\rangle^{*}\left|M_{j}\right\rangle .
\end{aligned}
$$

Comparing (38) and (41) leads to the conclusion that if $H$ is time-reversal symmetric, i.e. $H=\Theta H \Theta^{-1}$, then the Hamiltonian matrix is purely real in the $\left\{\left|M_{i}\right\rangle\right\}$ basis, while if $H$ is antisymmetric under $\Theta$, i.e. $H=-\Theta H \Theta^{-1}$, then the Hamiltonian matrix is purely imaginary.

Since $H$ is real in the $\left\{\left|M_{i}\right\rangle\right\}$ basis if and only if $H$ is time-reversal symmetric it follows that the unitary operator $U=\exp -i \tau H$ is self-transpose, i.e. $U=U^{T}$, if and only if $H$ is time-reversal symmetric. 
Now consider a time-dependent pulse described by the Hamiltonian $H_{P}(t)$. We assume that $H_{P}(t)$ is timereversal symmetric at all times, i.e. $H_{P}(t)=\Theta H_{P}(t) \Theta^{-1}$ for all $t$. The corresponding unitary evolution operator $U$ which evolves the system from time $t_{I}$ to $t_{F}$ can be written

$$
U=\lim _{N \rightarrow \infty} U\left(t_{N}\right) U\left(t_{N-1}\right) \cdots U\left(t_{2}\right) U\left(t_{1}\right)
$$

where

$$
U\left(t_{i}\right)=e^{-i \Delta t H_{P}\left(t_{i}\right)},
$$

with $\Delta t=\left(t_{F}-t_{I}\right) / N$ and $t_{1} \equiv t_{I}$ and $t_{N} \equiv t_{F}$.

Since $H_{P}\left(t_{i}\right)$ is time-reversal symmetric the above arguments imply $U^{T}\left(t_{i}\right)=U\left(t_{i}\right)$ when $U\left(t_{i}\right)$ is expressed in the time-symmetric basis $\left\{\left|M_{i}\right\rangle\right\}$. Thus, in this basis, we have

$$
\begin{aligned}
U^{T} & =\lim _{N \rightarrow \infty}\left(U\left(t_{N}\right) U\left(t_{N-1}\right) \cdots U\left(t_{2}\right) U\left(t_{1}\right)\right)^{T} \\
& =\lim _{N \rightarrow \infty} U^{T}\left(t_{1}\right) U^{T}\left(t_{2}\right) \cdots U^{T}\left(t_{N-1}\right) U^{T}\left(t_{N}\right) \\
& =\lim _{N \rightarrow \infty} U\left(t_{1}\right) U\left(t_{2}\right) \cdots U\left(t_{N-1}\right) U\left(t_{N}\right) .
\end{aligned}
$$

For a time-symmetric pulse $H_{P}\left(t_{i}\right)=H_{P}\left(t_{N+1-i}\right)$ and so $U\left(t_{i}\right)=U\left(t_{N+1-i}\right)$. This allows us to reverse the order of the operators in (46) which then implies

$$
U^{T}=U
$$

Thus if we write $U$ in terms of an effective Hamiltonian,

$$
U=e^{-i \tau H},
$$

the matrix elements of $H$ must be real in the timesymmetric basis. $H$ must therefore be time-reversal symmetric, i.e. $H=\Theta H \Theta^{-1}$.

To apply this theorem to the present problem we take the time-reversal operator for our two-electron system to be

$$
\Theta=e^{i \pi S_{A y}} e^{i \pi S_{B y}} K
$$

Here the antiunitary operator $K$ is defined so that when acting on a given state it takes the complex conjugate of the amplitudes of that state when expressed in the Hund-Mulliken basis defined in Sec. II. Note that this basis is constructed using the Fock-Darwin states, and if a magnetic field is present these states will be necessarily complex valued when expressed in the position basis. As defined here, the antiunitary operator $K$ only takes the complex conjugates of the amplitudes in the HundMulliken basis, it does not take the complex conjugate of the Fock-Darwin states themselves. Thus, if a magnetic field is present, $\Theta$ should be viewed as an effective timereversal symmetry operator. This is a technical point which does not affect any of our conclusions (provided the Zeeman coupling can be ignored — see below). The key property that we will need in what follows is that spin changes sign under time reversal, and it is readily verified that for our definition of $\Theta$,

$$
\Theta \mathbf{S}_{\mu} \Theta^{-1}=-\mathbf{S}_{\mu}
$$

for $\mu=A, B$ even in the presence of a magnetic field.

Under $\Theta$, the Hund-Mulliken basis states transform as follows,

$$
\begin{aligned}
\Theta\left|S_{i}\right\rangle & =\left|S_{i}\right\rangle \quad \text { for } i=1,2,3 \\
\Theta\left|T_{0}\right\rangle & =-\left|T_{0}\right\rangle \\
\Theta\left|T_{+}\right\rangle & =\left|T_{-}\right\rangle \\
\Theta\left|T_{-}\right\rangle & =\left|T_{+}\right\rangle
\end{aligned}
$$

The states $\left|S_{i}\right\rangle$ therefore form a time-symmetric basis for the singlet states. A time-symmetric basis for the triplet states is given by

$$
\begin{aligned}
\left|\tilde{T}_{0}\right\rangle & =i\left|T_{0}\right\rangle, \\
\left|\tilde{T}_{a}\right\rangle & =\frac{1}{\sqrt{2}}\left(\left|T_{+}\right\rangle+\left|T_{-}\right\rangle\right), \\
\left|\tilde{T}_{b}\right\rangle & =\frac{i}{\sqrt{2}}\left(\left|T_{-}\right\rangle-\left|T_{+}\right\rangle\right),
\end{aligned}
$$

all of which are eigenstates of $\Theta$ with eigenvalue +1 .

The matrix representation of $H_{H M}$ in the time-reversal invariant $\left|\tilde{T}_{0}\right\rangle,\left|S_{1}\right\rangle,\left|S_{2}\right\rangle$ basis is

$$
H_{H M}=\left(\begin{array}{ccc}
V & 0 & -2 P \\
0 & 0 & -2 t_{H} \\
-2 P & -2 t_{H} & U_{H}
\end{array}\right) \text {, }
$$

which is real, reflecting the effective time-reversal symmetry of $H_{H M}$. Note that this would not be the case if $H_{H M}$ included the Zeeman coupling of electron spins to an external magnetic field. While for typical field strengths the Zeeman coupling is small, ${ }^{2}$ for some parameters it can be comparable to the spin-orbit corrections considered here. If this is the case our conclusions following from effective time-reversal symmetry will no longer be valid. Of course in zero magnetic field exact time-reversal symmetry is guaranteed.

We now consider pulsing a time dependent $H_{H M}(t)$ adiabatically so that, according to the arguments of Sec. III, the resulting gate can be parametrized by an effective spin Hamiltonian $H$. Since at all times $t$ the Hund-Mulliken Hamiltonian is time-reversal symmetric, if the pulse itself is time symmetric, i.e. $H_{H M}(t)=$ $H_{H M}(-t)$ where we take the center of the pulse to be at $t=0$, then the above theorem implies that the effective spin Hamiltonian $H$ will also be time-reversal symmetric. Thus $H=\Theta H \Theta^{-1}$, and since $\Theta \mathbf{S}_{\mu} \Theta^{-1}=-\mathbf{S}_{\mu}$ this implies $H$ must be quadratic in the spin operators, and so $\alpha=0$. The resulting gate will therefore have the desired form (2).

For completeness we also consider here the case of time-antisymmetric pulsing. If $H_{P}(t)=-H_{P}(-t)$ then 

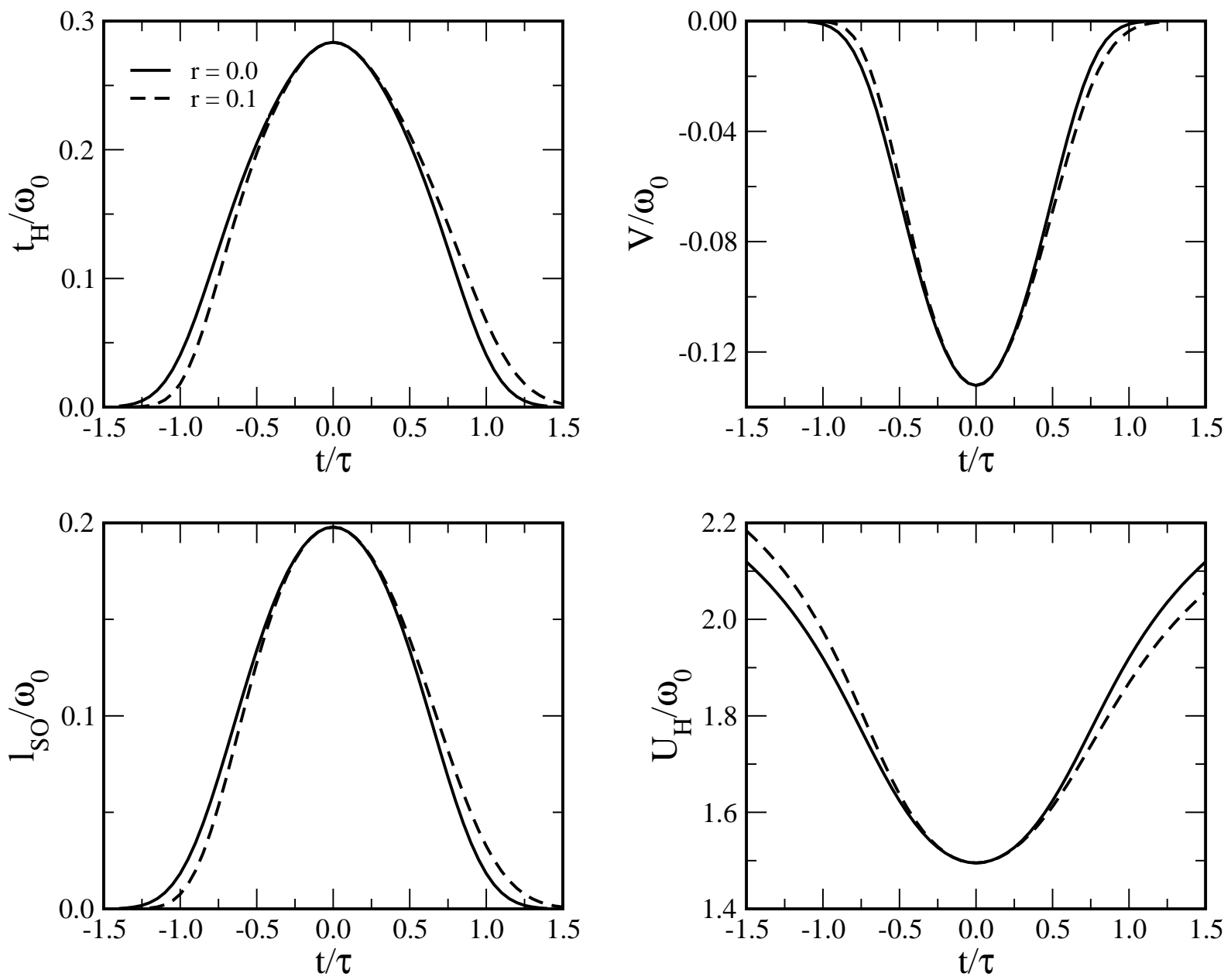

FIG. 2. Time dependence of matrix elements appearing in the Hund-Mulliken description of a double quantum dot when the displacement of the dots is varied according to (61) with $d_{0}=1$. Results are for GaAs parameters in zero magnetic field with $\hbar \omega_{0}=3 \mathrm{meV}$ and are plotted vs. the dimensionless quantity $t / \tau$ for two values of the time-asymmetry parameter, $r=0$ (solid line) and $r=0.1$ (dashed line).

$$
U(t)=e^{-i \Delta t H_{P}(t)}=e^{i \Delta t H_{P}(-t)}=U(-t)^{-1},
$$

and the resulting quantum gate is

$$
\begin{aligned}
U=\lim _{N \rightarrow \infty} & U\left(t_{1}\right) U\left(t_{2}\right) \cdots U\left(t_{N / 2}\right) \\
& \times U\left(t_{N / 2}\right)^{-1} \cdots U\left(t_{2}\right)^{-1} U\left(t_{1}\right)^{-1}=1 .
\end{aligned}
$$

The net effect of any time-antisymmetric pulse is thus simply the identity transformation.

\section{MODEL CALCULATIONS}

We have seen from symmetry arguments that timesymmetric pulsing of an axially symmetric Hamiltonian, such as $H_{H M}$ when $f_{D}$ and $f_{R}$ are constant, which is itself time-reversal symmetric at all times, will automatically produce a gate of the form (2), provided the pulse is adiabatic so that the initial and final states of the system are in the four-dimensional Hilbert space of two qubits.

It is natural to then ask what the effect of the inevitable deviations from time-symmetric pulsing will be on the resulting gate. To investigate this we have performed some simple numerical simulations of coupled quantum dots.

In our calculations, we imagine pulsing the dots by varying the dimensionless distance $d$ between them according to

$$
d(t)=d_{0}+\left(\frac{t}{\tau+r t}\right)^{2} .
$$

Here $d_{0}$ is the distance at the point of closest approach, $\tau$ is a measure of the pulse duration, and $r$ is a dimensionless measure of the time asymmetry of the pulse. This form describes the generic behavior of any pulse for times near the pulse maximum $(t=0)$. Note that for large $|t|$, and for $r \neq 0$, the distance $d(t)$ will saturate, and has a singularity for negative $t$. We have taken $r$ to be small enough so that the dots decouple long before this leads to any difficulty. 

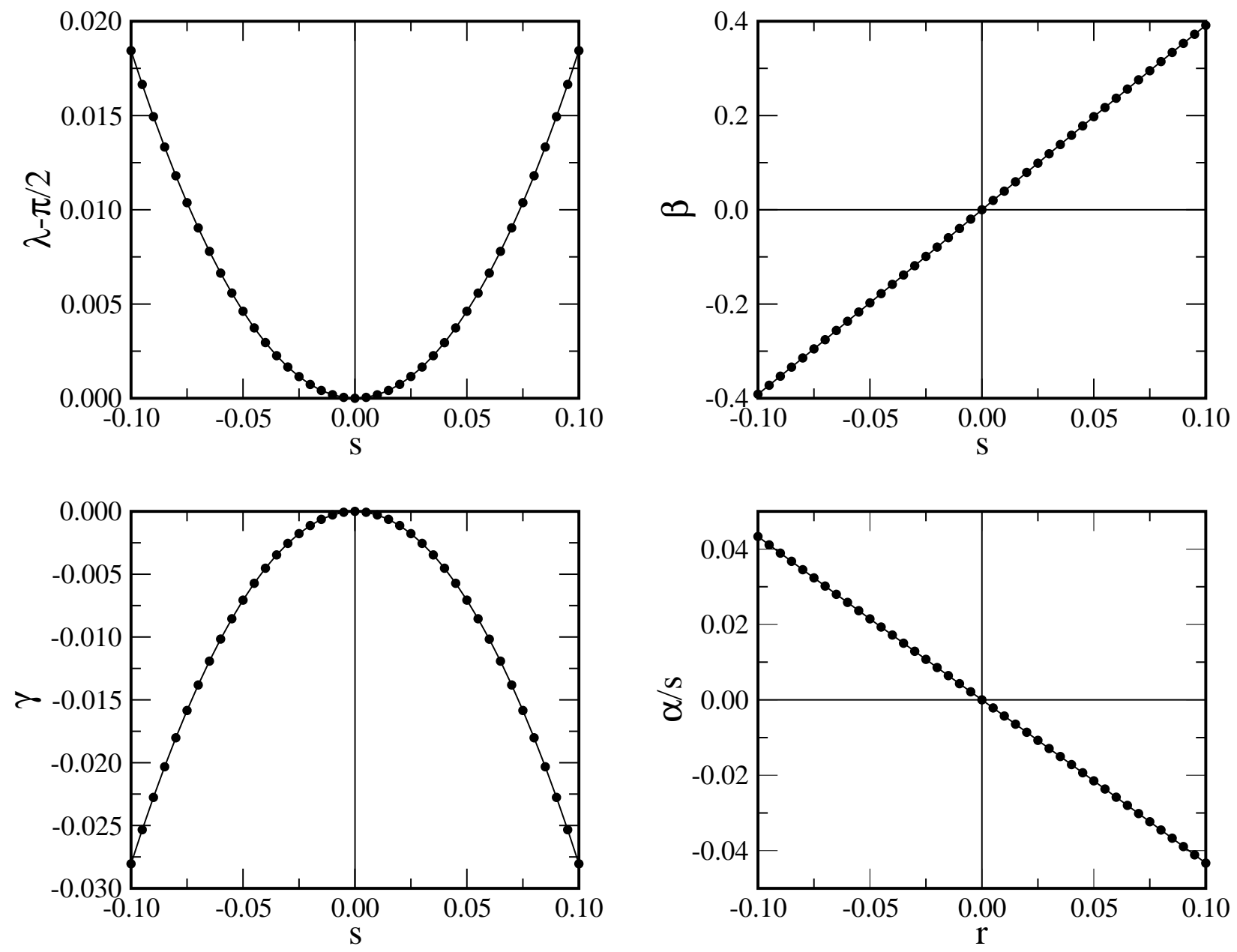

FIG. 3. Parameters appearing in the effective spin Hamiltonian derived from pulses depicted in Fig. 2. The parameters $\alpha$, $\beta$ and $\gamma$ are shown as functions of $s$ for the case $r=0$ (time-symmetric pulses). For $\alpha$ the quantity $\alpha / s$ is plotted vs. $r$. We have verified that the ratio $\alpha / s$ is essentially independent of $s$ for all values we have considered $(|s| \leq 0.1)$.

For our calculations, we work in zero magnetic field and take $\hbar \omega_{0}=3 \mathrm{meV}$ and $d_{0}=1$, corresponding to $a \simeq 20 \mathrm{~nm}$ at closest approach. The resulting time dependences of the parameters in $H_{H M}$ are shown in Fig. 2. Note that the spin-orbit matrix element plotted in this figure is $l_{S O}$, while the spin-orbit matrix element appearing in $H_{H M}$ is $\mathbf{P}=s l_{S O} \hat{z}$ where $s$ is the dimensionless measure of spin-orbit coupling introduced in Sec. II.

For a given pulse $H_{H M}(t)$ we integrate the timedependent Schrödinger equation to obtain the evolution operator $U$ for the full pulse. If the pulse is adiabatic then the matrix elements of $U$ which couple the singly occupied states $\left|S_{1}\right\rangle$ and $\left|T_{0}\right\rangle$ to the doubly occupied state $\left|S_{2}\right\rangle$ can be made negligibly small. ${ }^{5}$ The quantum gate is then obtained by simply truncating $U$ to the $4 \times 4$ matrix acting on the two-qubit Hilbert space. By taking the $\log$ of this matrix we obtain $\tau H=i \log U$ and thus the parameters $\lambda, \alpha, \beta, \gamma$. Note that when calculating $\log U$, there are branch cuts associated with each eigenvalue of $U$, and as a consequence $\tau H$ is not uniquely determined. We resolve this ambiguity by requiring that as the pulse height is reduced to zero and $U$ goes continuously to the identity that $\tau H \rightarrow 0$ without crossing any branch cuts.

We fix the pulse width $\tau$ by requiring that if we turn off spin-orbit coupling $(s=0)$ we obtain a $\lambda=\pi / 2$ pulse, i.e. a square-root of swap. For the parameters used here we find this corresponds to taking $\tau=23.9 / \omega_{0} \simeq 5 \mathrm{ps}$. We have checked that these pulses are well into the adiabatic regime. The magnitudes of the matrix elements coupling singly occupied states to the doubly occupied state $\left|S_{2}\right\rangle$ are on the order of $\left|\left\langle S_{1}|U| S_{2}\right\rangle\right| \sim 10^{-6}$ and $\left|\left\langle T_{0}|U| S_{2}\right\rangle\right| \sim s 10^{-6}$.

TABLE I. Symmetry properties of the pulse parameters $r$ and $s$, and gate parameters $\lambda, \alpha, \beta$ and $\gamma$ under parity $P$ and time reversal $T$.

\begin{tabular}{c|cc|cccc}
\hline \hline & $r$ & $s$ & $\lambda$ & $\alpha$ & $\beta$ & $\gamma$ \\
\hline $\mathrm{P}$ & + & - & + & - & - & + \\
$\mathrm{T}$ & - & + & + & - & + & + \\
\hline \hline
\end{tabular}


Once $\tau$ is fixed, there are two parameters characterizing each pulse, $s$ and $r$, and four parameters characterizing the resulting gate, $\lambda, \alpha, \beta$, and $\gamma$. The transformation properties of these parameters under parity $(P)$ and time reversal $(T)$ are summarized in Table I. These properties follow from the fact that (i) under time reversal $\mathbf{S}_{\mu} \rightarrow-\mathbf{S}_{\mu}$ and $r \rightarrow-r$, while $\mathbf{P}=s l_{S O} \hat{\mathbf{z}}$ is invariant, and (ii) under parity $\mathbf{S}_{A} \leftrightarrow \mathbf{S}_{B}$ and $\mathbf{P} \rightarrow-\mathbf{P}$, while $r$ is invariant. Note that, as defined in Sec. II, the parameter $s$ is positive. Here we allow $s$ to change sign when the direction of the vector $\mathbf{P}$ is reversed, thus under parity $s \rightarrow-s$.

These symmetry properties imply that if $s$ and $r$ are small, the parameters of the effective Hamiltonian will be given approximately by

$$
\begin{aligned}
\alpha & \simeq C_{\alpha} r s, \\
\beta & \simeq C_{\beta} s, \\
\gamma & \simeq C_{\gamma} s^{2}, \\
\lambda & \simeq \lambda_{0}+C_{\lambda} s^{2},
\end{aligned}
$$

where the coefficients should be of order 1 . For the pulses we consider here $\lambda_{0}=\pi / 2$.

The results of our calculations are shown in Fig. 3. Each point corresponds to a separate numerical run. The plots for $\lambda, \beta$ and $\gamma$ show their dependence on $s$ when $r=0$. The dependence of the parameter $\alpha$ on pulse asymmetry is shown by plotting $\alpha / s$ vs. $r$. For the $s$ values we have studied, up to $|s|=0.1$, the numerical results for $\alpha / s$ are essentially independent of $s$ for a given $r$. These results are clearly consistent with the above symmetry analysis.

Now consider carrying out a CNOT gate using the scheme reviewed in Sec. III. For this construction to work it is necessary that $\lambda=\pi / 2$. In our calculations we have fixed $\tau$ so that $\lambda=\pi / 2$ for $s=r=0$. Thus, when spin-orbit coupling is included

$$
\lambda \simeq \pi / 2+C_{\lambda} s^{2}
$$

In order to keep $\lambda=\pi / 2$ it will therefore be necessary to adjust the pulse width $\tau$ slightly to correct for spin-orbit effects.

The central result of this paper is summarized by the equation

$$
\alpha \simeq C_{\alpha} r s
$$

As shown in Sec. III, any nonzero $\alpha$ will lead to corrections to the CNOT construction. For time-symmetric pulses $r=0$ and these corrections will vanish. Equation (67) can then be used to estimate the errors due to any time asymmetry of the pulse, and to put design restrictions on the allowed tolerance for such asymmetry.

It is important to note that while the results presented here are for a specific model, all of the key arguments are based on symmetry and so are quite general. Given any time-reversal invariant two-qubit system with axial symmetry, if pulsed adiabatically in a time-symmetric way the resulting gate will have the form (2).

If the pulse is not axially symmetric, e.g. if the ratio $f_{D} / f_{R}$ varies during the pulse, then time-symmetric pulsing will still restrict the resulting gate to be invariant under time reversal. Thus, up to an irrelevant overall phase, this gate will necessarily have the form

$U=\exp -i \lambda\left(\mathbf{S}_{A} \cdot \mathbf{S}_{B}+\boldsymbol{\beta} \cdot \mathbf{S}_{A} \times \mathbf{S}_{B}+\mathbf{S}_{A} \cdot \boldsymbol{\Gamma} \cdot \mathbf{S}_{B}\right)$.

Here $\boldsymbol{\Gamma}$ is a symmetric tensor which will, in general, deviate from the axial form of the $\gamma$ term in (2) leading to corrections to the CNOT construction. However, because $\boldsymbol{\Gamma}$ is even under parity it will still be second order in spin-orbit coupling, ${ }^{12}$ and thus the deviations from (2) will also be second order. We conclude that even in the absence of axial symmetry, the corrections to the CNOT construction will be second order in spin-orbit coupling, rather than first order.

\section{CONCLUSIONS}

In this paper we have studied spin-orbit corrections to exchange-based quantum gates, emphasizing symmetry arguments. In particular, we have shown that adiabatic time-symmetric pulsing of any Hamiltonian which (i) describes two well defined spin-1/2 qubits at the beginning and end of the pulse, (ii) is time-reversal symmetric at all times during the pulse, and (iii) is axially symmetric in spin space with a fixed symmetry axis, will automatically produce a gate of the form (2). Together with single qubit rotations, for $\lambda=\pi / 2$ this gate can then be used in a simple CNOT construction. This result is quite general.

As a specific example we have studied a GaAs double quantum dot system within the Hund-Mulliken approximation. In this approximation spin-orbit coupling enters as a small spin precession when an electron tunnels between dots. If the direction of this precession axis is constant throughout the pulse the resulting gate will be axially symmetric and have the form (29). The deviation of this gate from the desired gate (2) is then characterized by a single dimensionless parameter $\alpha$ which spoils the CNOT construction. Using symmetry arguments, as well as numerical calculations, we have shown that $\alpha \simeq C_{\alpha} s r$ where $s$ and $r$ are, respectively, dimensionless measures of spin-orbit coupling and time asymmetry of the pulse. Thus time-symmetric pulsing $(r=0)$ ensures the anisotropic corrections will have the desired form.

In any system without spatial inversion symmetry, spin-orbit coupling will inevitably lead to anisotropic corrections to the exchange interaction between spins. According to current estimates, ${ }^{18}$ fault-tolerant quantum computation will require realizing quantum gates with an accuracy of one part in $10^{4}$. Thus, even if spin-orbit 
coupling is weak, the design of any future quantum computer which uses the exchange interaction will have to take these anisotropic corrections into account. We believe the symmetry based analysis presented in this paper provides a useful framework for studying these effects.

\section{ACKNOWLEDGMENTS}

DS and NEB acknowledge support from the National Science Foundation through NIRT Grant No. DMR0103034. DPDV is supported in part by the National Security Agency and the Advanced Research and Development Activity through Army Research Office contract number DAAD19-01-C-0056. He thanks the Institute for Quantum Information at Cal Tech (supported by the National Science Foundation under Grant. No. EIA0086038) for its hospitality during the initial stages of this work. DL thanks Swiss NSF, NCCR Nanoscience, DARPA and ARO.

\footnotetext{
${ }^{1}$ D. Loss and D.P. DiVincenzo, Phys. Rev. A 57, 120 (1998).

${ }^{2}$ G. Burkard, D. Loss, and D.P. DiVincenzo, Phys. Rev. B 59, 2070 (1999).
}

${ }^{3}$ G. Burkard, D. Loss, D.P. DiVincenzo, and J.A. Smolin, Phys. Rev. B 60, 11404 (1999).

${ }^{4}$ X. Hu and S. Das Sarma, Phys. Rev. A 61, 062301 (2000).

${ }^{5}$ J. Schliemann, D. Loss and A.H. MacDonald, Phys. Rev. B 63, 085311 (2001).

${ }^{6}$ D. Bacon, J. Kempe, D.A. Lidar, and K.B. Whaley, Phys. Rev. Lett. 85, 1758 (2000).

${ }^{7}$ D.P. DiVincenzo, D. Bacon, J. Kempe, G. Burkard and K.B. Whaley, Nature 408, 339 (2000).

${ }^{8}$ G. Burkard and D. Loss, Phys. Rev. Lett. 88, 047903 (2002).

${ }^{9}$ L.-A. Wu and D. Lidar, Phys. Rev. A 66, 062314 (2002).

${ }^{10}$ K.V. Kavokin, Phys. Rev. B 64075305 (2001); condmat/0212347.

${ }^{11}$ L.P. Gor'kov and P.L. Krotkov, Phys. Rev. B 67, 033203 (2003).

12 N.E. Bonesteel, D. Stepanenko, and D.P. DiVincenzo, Phys. Rev. Lett. 87, 207901 (2001).

13 G. Dresselhaus, Phys. Rev. 100, 580 (1955).

${ }^{14}$ M. I. Dyakonov and V. Y. Kachorovskii, Sov. Phys. Semicond. 20, 110 (1986).

${ }^{15}$ E. L. Rashba, Fiz. Tv. Tela (Leningrad) 2, 1224 (1960) [Sov. Phys. Solid State 2, 1109 (1960)]; Y.A. Bychkov and E.I. Rashba, J. Phys. C 17, 6039 (1984).

16 J. Schliemann, J.C. Egues, and D. Loss, Phys. Rev. Lett. 90, 146801 (2003).

${ }^{17}$ For an excellent discussion of time-reversal symmetry see, "Quantum Mechanics", K. Gottfried, Addison-Wesley, 1989, pp. 314-322.

${ }^{18}$ D. Aharonov and M. Ben-Or, quant-ph/9906129. 